\title{
サプライネットワーク環境下における市場の概念に 基づいた企業戦略策定に関する研究*
}

\author{
オパヂジ ジャイェオラ フェミ†・貝原 俊也
}

\author{
A Study on Market-based Strategic Procurement Planning \\ in Convergent Supply Networks*
}

\author{
Jayeola Femi OpAdisi ${ }^{\dagger}$ and Toshiya KAIHARA ${ }^{\ddagger}$
}

\begin{abstract}
We present a market-based decentralized approach which uses a market-oriented programming algorithm to obtain Pareto-optimal allocation of resources traded among agents which represent enterprise units in a supply network. The proposed method divides the network into a series of Walrsian markets in order to obtain procurement budgets for enterprises in the network. An interaction protocol based on market value propagation is constructed to coordinate the flow of resources across the network layers. The method mitigates the effect of product complementarity in convergent network by allowing for enterprises to hold private valuations of resources in the markets.
\end{abstract}

\section{Introduction}

The market-based approach to supply network planning requires enterprise units in the supply network to focus their strategies on remaining competitive in the network [1,2]. Procurement planning is one of the main activities at the strategic planning level of the supply network. Since organizations in a supply network are legally independent entities and often have competing goals, it is necessary to device a way in which these enterprise units can generate independent strategic plans which will mutually benefit participating parties. One way of achieving this is by modeling the supply network as a pure-competition market [3] in which price mechanisms will determine how resources are allocated to participants in the market. However, one of the major drawbacks of this approach is the very strong assumptions on which the pure-competition market is based; this includes

\footnotetext{
* 原稿受付 2008 年 5 月 28 日

†神戶大学 大学院 自然科学研究科 Grad. School of Sci. and Tech., Kobe University; 1-1 Rokkodai, Nada ward, Kobe city, Hyogo 657-8501, JAPAN

¥ 神戶大学 大学院 工学研究科 Grad. School of Engineering, Kobe University; 1-1 Rokkodai, Nada ward, Kobe city, Hyogo 657-8501, JAPAN
}

Key Words: multi-agent system, supply network, marketoriented programming, resource allocation. the assumption of gross substitionality of market resources [4]; this means that resources in the market are similar in usage and therefore an increase in preference for one will mean a reduction in preference for others, i.e., the value produced when this resources are combined is less than the sum of the individual values of each resource. In a serial network where value creation involves the transformation of a single type of production resource to another at each production layer of the supply network, this assumption is plausible especially since all the enterprise units at each production phase are producing alternative products which may be consumed by any unit in the succeeding layer. For a convergent supply network however, the assumption becomes less credible since production of a resource involves the combination of more than one type of input resource. A convergent network scenario thus results in a market with complementary resources as demand and supply for these resources are interconnected.

In this study, we create stage-wise computation that uses pure-competition market mechanism for resource allocation while assuming that the autonomy of enterprise units represent their responsibility for decision making as it affects their preference for suppliers of a particular resource and their private budgets for 
complementary resources. While a number of algorithms have been constructed to find computable general equilibrium $[4,5]$, our contribution lies in applying the concept to generate procurement plans for multilayered convergent network intended to preserve autonomy of enterprises in the network. We focus on the protocol that generates procurement budgets for enterprises in the supply network making use of quantified supplier preferences and input resource valuations assumed to already have been computed by enterprise agents. Using the market-based approach, a convergent supply network is assumed to be a single virtual enterprise made up of different markets for all the market resources, the combination of which ultimately leads to final product consumption in the network. As defined in [6], "A virtual enterprise is a temporary alliance of enterprises that come together to share core competencies and resources in order to better respond to business opportunities and whose cooperation is supported by computer networks". In order words, the business enterprises are only connected to one another in a virtual domain.

\section{Strategic Planning Problem}

The convergent supply network treated in this work is an agglomeration of different markets which make up an industry, e.g. automobile industry, personal computer industry, etc. In this model, estimated demands from the consumer markets represent the investments into the supply network. Financial investments move upstream while products move downstream. Fig. 1 illustrates the convergent supply network considered in this work.

In Fig. 1, there are two types of resources - the primary production resources (capital and labour) and the supplier market resources. The supply network is divided into layers from the upstream to the downstream; each of these layers consists of markets represented by the circles. The markets contain enterprise units producing the same kind of products and they are represented by the different shapes in the circles. The investment layer is made up of investment agents that act as consumers of the final products of the supply network. Also, all the markets except markets in the most upstream layer require resources from markets in their preceding layer and supply markets in their succeeding layer. The following are the assumptions for the supply network:

- Every enterprise unit in a market is autonomous.

- Each enterprise unit produces one class of prod-

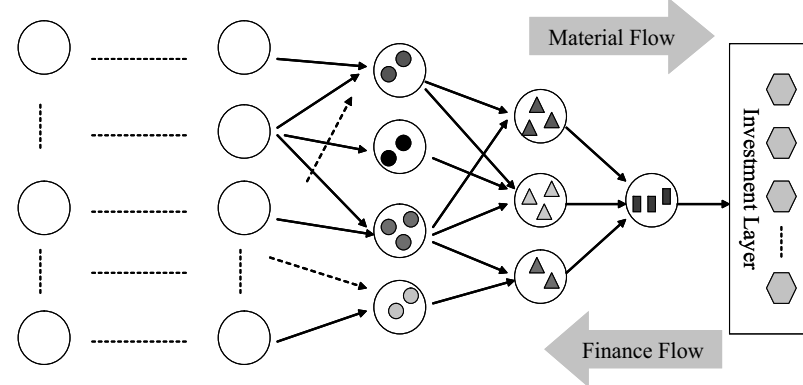

Fig. 1 Convergent supply network

uct: at the strategic level, enterprise resources are aggregated.

- For every input an enterprise unit requires from a market there are more than one possible suppliers.

- An enterprise unit has private valuation of what percentage of its resources it is willing to invest in a particular market.

It is required to find a Pareto-optimal allocation of resources for all the enterprises trading in a given market. This means, that for every market in which an enterprise is trading, its allocation should be such that it cannot receive a better allocation from any supplying enterprise in that market without any other consuming enterprise getting a sub-optimal allocation. In modeling this problem, we consider the supply network as a Virtual Enterprise (VE). In this case, the presence of the enterprise in the network is only recognized by their participation in economic activities in the network. The list of symbols used is defined as follows:

Layer $S:\{Q, M\} ; Q=$ investment layer

$M=$ set of value transformation layers

$P:\left\{p_{y}, p_{K}, p_{L}, p_{1}, p_{2}, \ldots, p_{r}\right\}$

$Q:\left\{q_{1}, q_{2}, \ldots, q_{r}\right\} ; q_{i}=$ investment agent $i$

$M:\left\{M_{1}, M_{2}, \ldots, M_{l}\right\} ; M_{i}=$ set of markets in layer $i$

$G:\left\{g_{1}, g_{2}, \ldots, g_{r}\right\} ; G=$ set of resources in VE network

$g_{i}=$ resource traded in market $i$

$F:\left\{f_{11}, f_{12}, \ldots, f_{m n}\right\} ; F=$ set of enterprises in VE network

$f_{i j}=$ enterprise $i$ in layer $j$; $f_{i j} \in M_{j k} \subseteq M_{j}$

$f_{i j}:\left\{a_{o}^{i j}, a_{g_{1, j+1}}^{i j}, \ldots, a_{g_{\left|M_{j+1}\right|, j+1}^{i j}}^{i j}\right\}$

$a_{o}^{i j}=$ production agent for $f_{i j}$

$a_{g_{c, j+1}}^{i j}=$ procurement agent for market resource $g_{c}$ in layer $(j+1)$

All the enterprises must maximize the amount of 
inputs they are able to source from their suppliers subject to budgetary constraints:

$$
\max R_{f_{i j}}:\left\{g_{1}^{i j}, g_{2}^{i j}, \ldots, g_{r}^{i j}\right\},
$$

where $g_{t}^{i j}=\sum_{t=1}^{n} x_{t s}^{i j} ; 1 \leq t \leq r ;(r \in N)$, s.t.

$$
\begin{aligned}
b_{i j} & =p_{K} \cdot K_{i j}+p_{L} \cdot L_{i j}, \\
b_{i j} \geq \sum_{t=1}^{r} p_{t} g_{t}^{i j} & \\
b_{i j} & =p_{y} y_{i j}, \\
R_{f_{i j}} & =\text { set of inputs of enterprise } f_{i j} \\
b_{i j}= & \text { budget of enterprise } f_{i j} \\
K_{i j} & =\text { total capital budget allocated to } f_{i j} \\
L_{i j} & =\text { total labour budget allocated to } f_{i j} \\
g_{t}^{i j}= & \text { quantity of input } g_{t} \text { allocated to } f_{i j} \\
y_{i j}= & \text { quantity of output supplied by } f_{i j} \\
x_{t s}^{i j}= & \text { quantity of input } g_{t} \text { sourced from supplier } s \\
& \text { by } f_{i j} \\
n= & \text { number of suppliers in layer }(j+1) \text { supplying } \\
& \text { resource } g_{t} \text { to } f_{i j} \\
N= & \text { set of natural numbers }
\end{aligned}
$$

\section{Solution Methodology}

From the description above, every enterprise is an economic unit which employs the services of one production agent and one or more procurement agents. The production agent trades in the output market of the enterprise and the procurement agents trade in the input markets. The procurement agents are responsible for securing the necessary inputs the enterprise needs to produce its output. The production agent on the other hand has to secure as much primary production resources as possible from prospective consumers so that the enterprise can endow its procurement agents for trade in the input market. Figs. 2 - 4 give pictorial representations of an enterprise unit and its agents.

\subsection{Enterprise Unit}

An enterprise belongs to a market in a particular layer of the supply network. It is a unit in the network responsible for creating a new resource by transforming a set of input resources. Interaction among enterprises is via a set of trading agents that are instantiated when value creation activities are triggered in the network. An enterprise unit has a private valuation of its input resources with which it allocates primary production resources to its procurement agents. Fig. 2 shows a schematic of the enterprise unit.

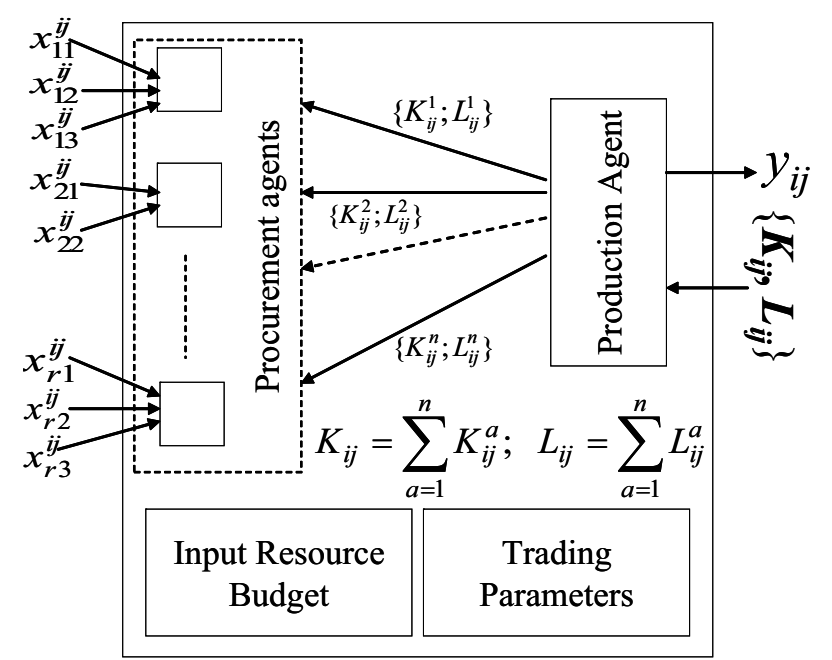

Fig. 2 Example of an enterprise unit

To Procurement Agents

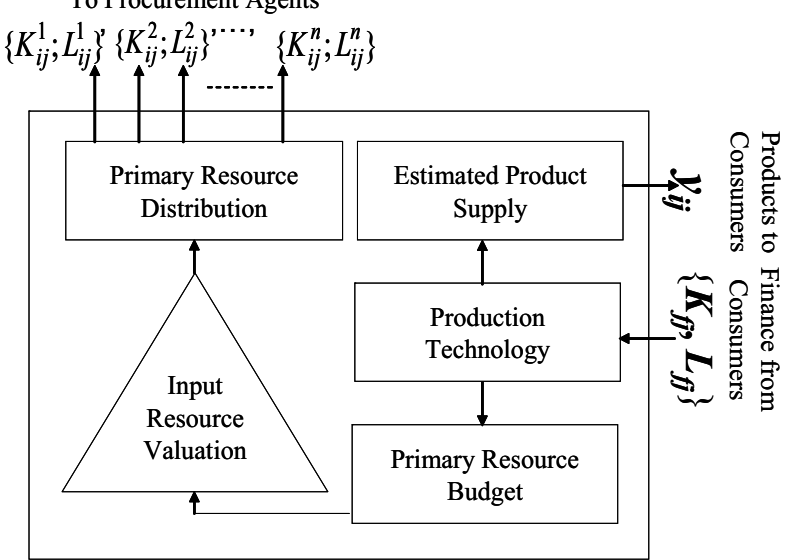

Fig. 3 Production agent of enterprise unit

\subsection{Production Agent}

The production agent of an enterprise represents the enterprise in the sales market. It acts as the supplier of an enterprise output. The schematic of the production agent is in Fig. 3.

- The agent receives primary production resources (capital and labour) from prospective consumers as rent to produce its output resource.

- It reports its allocation of capital and labour to the enterprise as its share from the market.

- The production agent also distributes the primary resource allocations among the procurement agents of the enterprise based on an internalized input valuation ratio of that enterprise unit.

\subsection{Procurement Agent}

A procurement agent is responsible for securing input resources needed by an enterprise from an input market in the preceding layer.

- Each of the input resources of an enterprise can be provided by more than one supplier. 


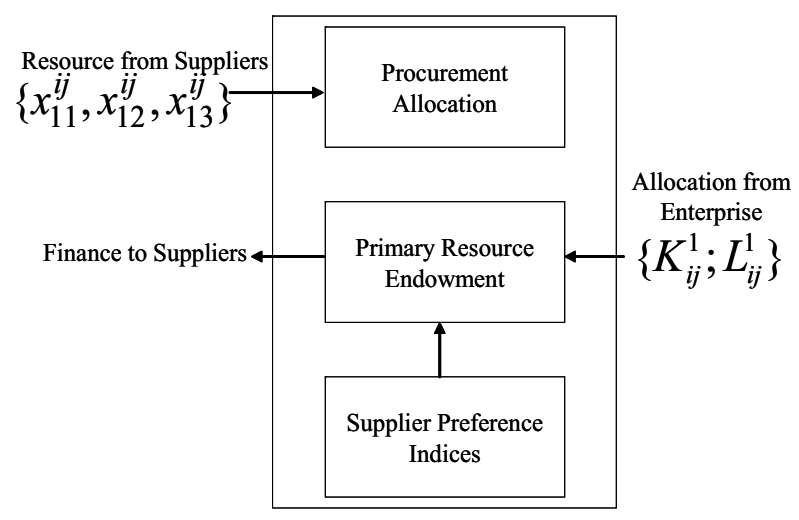

Fig. 4 Procurement agent of enterprise unit

- A procurement agent has quantified private preference for every possible supplier.

- Capital and labour endowments of a procurement agent are provided by the enterprise production agent.

- Capital and labour endowments of the procurement agent are exchanged for needed input resource.

\section{Market Value Propagation Proto- $\operatorname{col}(\mathrm{MVPP})$}

The idea of market value propagation stems from the fact that enterprises in a supply network focus mainly on markets in their immediate neighbourhood, i.e., the layers preceding and succeeding them. Therefore, their goal will be how to select their strategies so as to remain competitive in these markets. Another important issue is that the total amount of revenue accrued to enterprises in each layer depends on the total amount of investment into the market by the consumer layer. The result is that, financial resources flow in the form of capital and labour upstream while materials flow from upstream to downstream. The competition for resources by enterprises in the same layer but not in the same market also affects the distribution of both financial and material resources.

In the market value propagation protocol, distribution of market resources is done sequentially along the supply network. This is illustrated in Fig. 5 .

The MVPP algorithm is listed as follows:

Step 1 Get number of value creation layers $=m$.

$\overline{\text { Step } 2}$ Set current layer to final product market (FPM) layer $=1$.

Step 3 Set consumers to consumer layer: investment agents.

Step 4 Set suppliers to current layer: production agents. Step 5 Execute Market-Oriented programming (MOP)

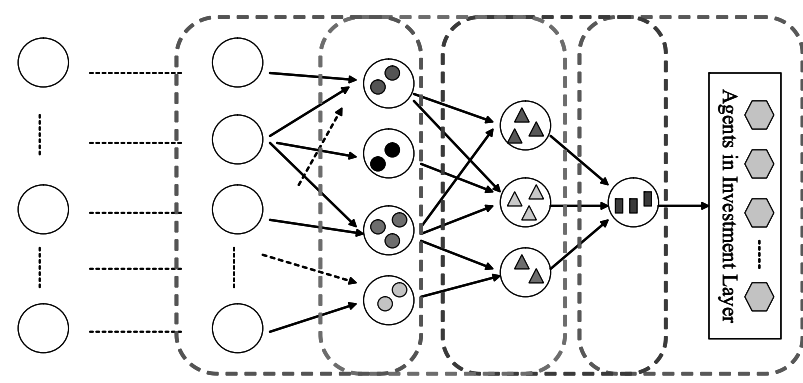

Fig. 5 Virtual market interactions among network layers algorithm between consumers and suppliers.

Step 6 Determine allocation of primary resources $(K$ and $L$ ) for all Production agents in current layer.

Step 7 Current layer: production agents distribute $K$ and $L$ allocation to their corresponding procurement agents.

Step 8 If current layer is equal to $m$ go to Step 11 .

$\overline{\text { Step } 9}$ Set consumers to current layer: procurement agents.

Step 10 Increment current layer; go to Step 4.

$\overline{\text { Step } 11}$ End Protocol.

The MVPP algorithm listed above describes the movement and utilization of primary production resources by enterprise units for value creation. All primary resources (capital $K$ and labour $L$ ) used in the supply network are provided by the investment layer in the form of market investment. The total investment into the supply network is redistributed at each layer. The process of redistribution of market investment is executed by the MOP algorithm[5] described in section 4.1. Movement of resources thus is in two directions; primary resources rented out by consumers move from the investment layer to the final product layer from where it is used to procure input production resources from the layer preceding it. Enterprises in this preceding layer also use primary resources distributed to them to procure resources from the layer preceding it; this process continues until the last layer in the network is reached, hence the name, market value propagation protocol. Just as primary resources are propagated from downstream to upstream, so also are production input resources propagated from upstream to downstream in the supply network. Competitive markets are created at each propagation stage and resources are redistributed to enterprises through the MOP algorithm.

In Step 5 of the MVPP, the MOP algorithm to be executed depends on the Walrasian market[3] concept. This algorithm has been found to be faster 
in reaching a Pareto-optimal allocation of resources among traders in a competitive market than some other competitive market algorithms[9]. The performance of this algorithm as compared with the Scarf algorithm and Price tantonnement algorithm also used in competitive market equilibrium analysis is shown in [7] and [8].

\subsection{MOP in Pure Competitive (Wal- rasian) Market}

A market is any arrangement that facilitates the exchange of resources by trading agents who either seek to maximize their utility from the consumption of such resources or profits from the supply of the resources. For the ease of exchange, a price system is defined which allocates a nonnegative number to market resources in order to determine their relative value. The term competitive market denotes the existence of contention for resources among traders in that market. In order for trading to take place, the total amount of goods demanded in the market equal the supply. In a pure competition market, this assumption is extended further to mean quantity supplied being equal to that which is demanded. With the existence of a price system, such an equilibrium point can be found if it exists by adjusting the prices of goods in response to market demand and supply. MOP considers a unique type of competitive market the Walrasian market. The model guarantees the existence of equilibrium in a market with multiple goods which are grossly substitutional.

The Walrasian market guarantees the existence of equilibrium in pure-competition market with multiple goods which are grossly substitutional. The market is modeled as follows:

$$
g_{i} \in G ; 1 \leq i \leq k,
$$

where $G$ is a set of goods in the market.

$$
\begin{aligned}
& p_{i}\left(g_{i}\right) \in P ; P \text { is price vector, } \\
& e_{i}^{j} \subset E ; 1 \leq i \leq k, \\
& e_{i}^{j} \text { endowment of trader } j \text { on resource } i .
\end{aligned}
$$

Given a set of $n$ consumers and $m$ suppliers in an economy with $k$ goods, budget of a consumer $c$ is represented as:

$$
B^{c}=\sum_{i=1}^{k} p_{i} e_{i}^{c}+\pi_{c} .
$$

$\pi_{c}=$ profit consumer for renting out its endowment.

The optimization problem for consumer $c$ is:

$$
\max U^{c}\left(g_{1}, g_{2}, \ldots, g_{k}\right)
$$

where $U^{c}$ is utility function subject to budget constraint:

$$
\sum_{i=1}^{k} p_{i} g_{i}^{c} \leq B^{c}
$$

and a non-negative price constraint:

$$
p_{i} \geq 0 ; \text { for all } p_{i} \in P ; 1 \leq i \leq k .
$$

The utility function is represented as a Cobb-Douglas function with constant returns-to-scale[3]; this function defines the value of each consumed resource in terms of the preference index of the consumer:

$$
U^{c}=\prod_{t=1}^{k} g_{t}^{\alpha_{t}^{c}} ; \sum_{t=1}^{k} \alpha_{t}^{c}=1,
$$

$\alpha_{t}^{c}=$ preference of consumer for resource $g_{t}$. Using the Lagrange multiplier approach, an optimal bidding function is obtained for the consumer bidding problem as:

$$
g_{t}^{c}\left(p_{t}\right)=\frac{\alpha_{t}^{c} B^{c}}{p_{t}} .
$$

The rationality of suppliers dictates that they seek to maximize profit. Therefore, the constrained optimization problem of a supplier $s$ is:

$$
\begin{aligned}
& \max \pi_{s}=p_{s} g_{s}-\sum_{\substack{i=1 \\
i \neq s}}^{k} p_{i} g_{i}, \\
& \pi_{c}=\delta_{c} \sum_{s=1}^{m} \pi_{s},
\end{aligned}
$$

where $\pi_{s}$ is profit of supplier $s$ and $\delta_{c}$ is profit sharing ratio of $c$ subject to technology of supplier $s=T^{s}$ :

$$
\begin{aligned}
& T^{s}\left(g_{1}, g_{2}, \ldots, g_{s-1}, g_{s+1}, \ldots, g_{k}\right)=g_{s} \\
& g_{s}=\Gamma \prod_{t=1 \neq s}^{k} g_{t}^{\beta_{t}^{s}} ; \sum_{t=1}^{k} \beta_{t}^{s}=1
\end{aligned}
$$

$g_{s}$ is output of supplier $s$,

$$
g_{i} \geq 0 ; \text { for all } g_{i} \in G ; 1 \leq i \leq k .
$$

$\beta_{t}^{s}=$ technology index of supplier for resource $g_{t}$

$\Gamma=$ production scale of supplier $g_{t}$

Using the Lagrange multiplier approach, the optimal bidding function for a supplier is obtained as:

$$
g_{t}^{s}\left(p_{t}\right)=\left(\frac{p_{t}}{\Gamma \beta_{t}^{s} p_{s}}\right)^{\left(\frac{1}{\beta_{t}^{s}-1}\right)} .
$$

The equilibrium point is defined as when demand is equal to supply for all market resources, 


$$
\sum_{i=1}^{m} d_{g_{i}}=\sum_{i=1}^{m} s_{g_{i}} ; \text { for all } g_{i} \in G
$$

\section{Experiment and Results}

\subsection{Target Supply Network}

Fig. 6 shows a target network used for simulating MVPP in a virtual enterprise environment.

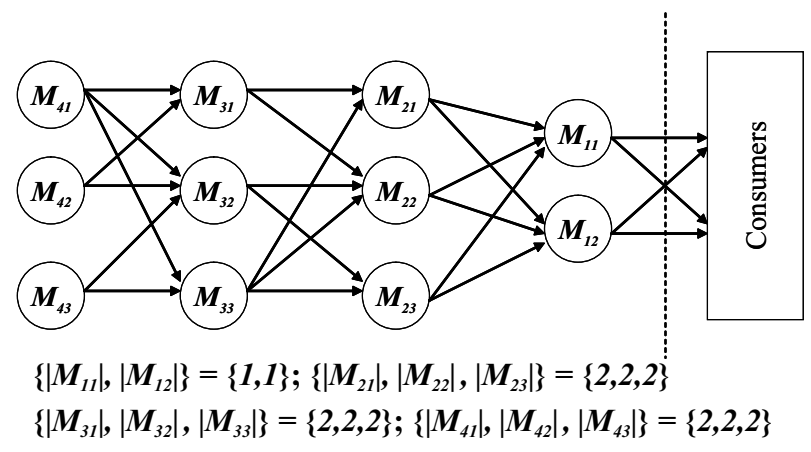

Fig. 6 Target supply network

The following experimental data were used in the simulation: Endowment and preference index matrices of consumers in investment layer:

$$
\begin{aligned}
& \{K, L\}^{T}=\left(\begin{array}{lllll}
100 & 100 & 100 & 100 & 100 \\
100 & 100 & 100 & 100 & 100
\end{array}\right), \\
& \left\{\alpha_{\mathbf{g} 11}, \alpha_{\mathbf{g} 12}\right\}^{T}=\left(\begin{array}{lllll}
0.50 & 0.30 & 0.60 & 0.80 & 0.40 \\
0.50 & 0.70 & 0.40 & 0.20 & 0.60
\end{array}\right) .
\end{aligned}
$$

The data for the enterprise units are shown in Fig. 7. The columns represent data for each consumer in the investment layer and each enterprise in the markets of the virtual enterprise. The blank columns denote value zero.

\subsection{Experimental Results}

First we present a result from price variation in the Walrasian markets during value propagation. In Fig. 8, it can be seen that equilibrium is reached for all the resources in the market, i.e., a point is reached when no trading agent is willing to change its bid for all of the market resources. This is the same for all the other Walrasian markets.

Next we show an example of allocation of primary production resources among the enterprise units in the resource transformation layers. Fig. 9 shows that the total allocation of primary production resources in a particular market are equal; the same holds for all the other markets. This means that financial value is completely propagated from one layer to the next in the upstream direction. Also, the total amount of capital and labour in each layer is equal to the total that is invested by the consumers in the investment layer, which tallies with the assumption of conservation of value in the perfect competition market. Distribution of an input resource in a layer depends on the endowments of the procurement agents in that layer. The endowment of procurement agents represents the purchasing power of an enterprise in the market where the resource is traded. Hence, one of the most important strategic decisions an enterprise will take has to do with its private valuations of resources it needs for production. In this work, we assume certain constant values for these private valuations, however, the determination of this private valuations is important so that there will be a relative balance in the amount of raw materials planned for over the strategic period. When tallied, the total supplied of all resources in each layer is equal to the total demanded in the succeeding layers downstream. This also confirms the assumption of conservation of products in a perfect competition market.

Fig. 10 represents the allocation of resources to agents in a layer of the network. This form of graph was obtained for all the other layers in the network. The allocation of resources is determined by the bidding function as defined in equation (9). This shows that the allocation of a resource to an agent depends on its preference index for that resource, its budget as well as the price of the resource. The allocation in Fig. 10 therefore is a function of the endowment matrix, the preference matrix in section $\mathbf{5 . 1}$, the price graph in Fig. 8 and profit sharing ratio defined by the market. For consumers 2, 3 and 4 , the allocation of resources partially correlates to their preference values, but this is not the same for consumers 1 and 5 . The reason for this can be found in the prices of the two resources; with resource 1 having a lower price than resource 2 , therefore even when the preferences are almost the same, resource 1 is clearly more preferred. Even for consumer 2 where the preference index indicates a much higher value for resource 2 , the difference in allocation is not as large owing to the prices of the resources. It can also be seen that consumer 1 has a larger allocation of resource 1 than consumer 4 even though consumer 4 has a higher preference value. This is due to consumer 1 having a larger budget because of an assumed larger profit sharing ratio [see equations (4) and (11)]. This phenomenon has been confirmed for all the other layers in the supply network.

Lastly, the output estimates for all the enterprises in the VE environment are shown in Fig. 11. Using 


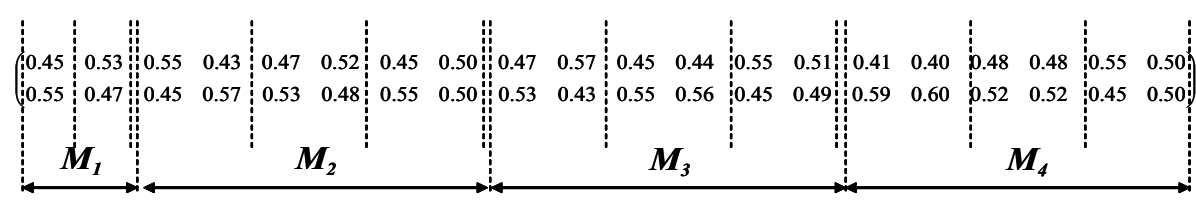

(a) Technology indices of enterprise units

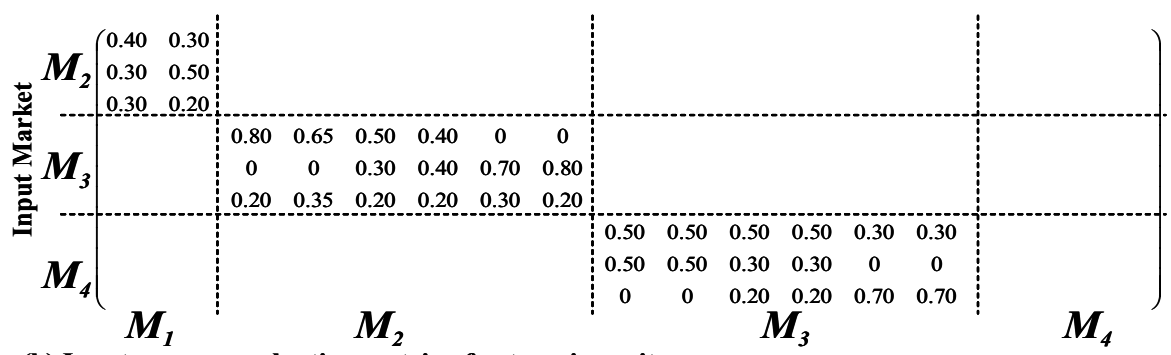

(b) Input resource valuation matrix of enterprise units

Fig. 7 Data for enterprise units

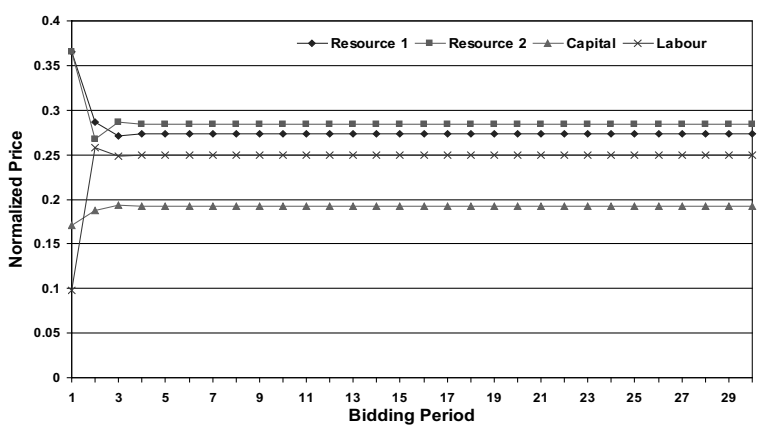

Fig. 8 Price fluctuation in market 1: Investment layer and Layer 1

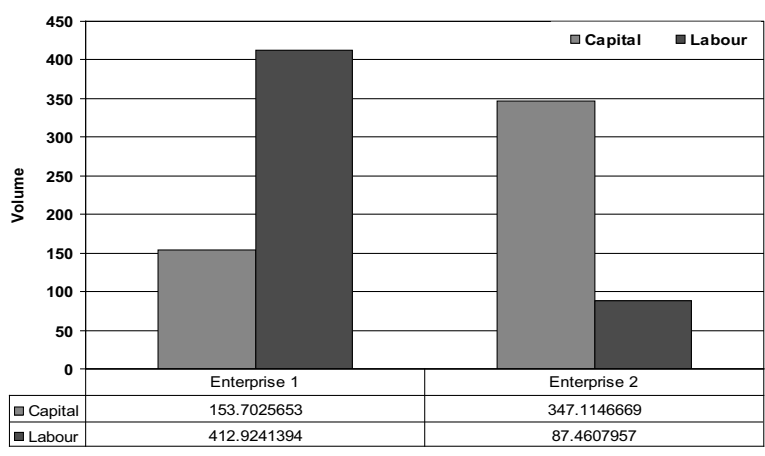

Fig. 9 Capital and Labour allocation in Market 1

enterprises in layer 1 of Fig. 11 as an example and comparing it with the resource allocation graph of Fig. 10, it can be seen that the total demand of resources 1 and 2 by consumers in the investment layer shown in Fig. 10 are approximately equal to the supply of the same resources in layer 1 as depicted in Fig. 11. This confirms the competitive equilibrium condition in the market as defined in equation (16) (the same holds for all the other layers). The output quantities shown in Fig. 11 are derived from the production function in equation (13) while the quantities

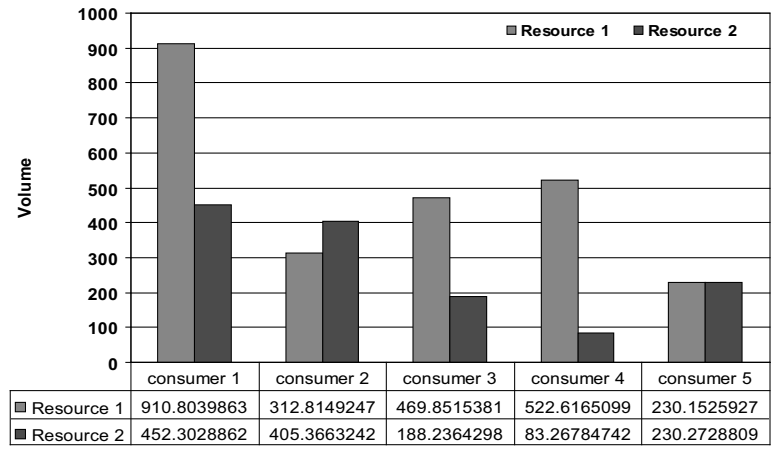

Fig. 10 Resource allocation in investment layer

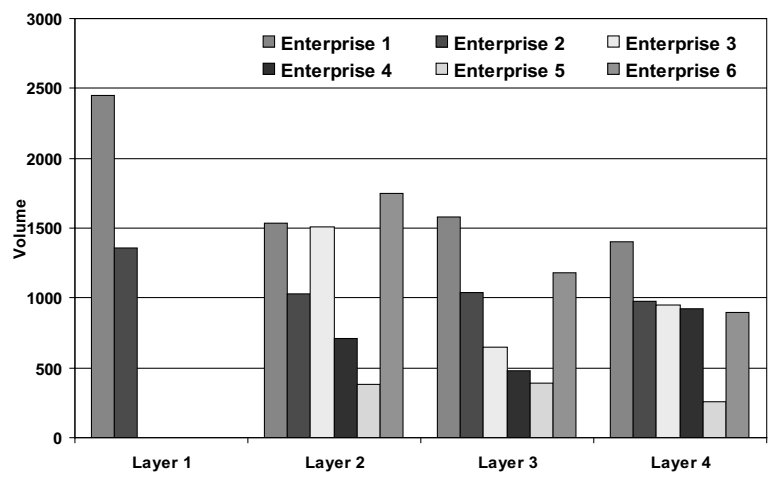

Fig. 11 Output estimates for enterprises

of input resources for the enterprises are derived from the bidding function in equation (15). It can be seen from equation (13) that with a given larger production scale, enterprise 1 produces more than enterprise 2. Furthermore, the outputs are a function of the amount of input resources (capital and labour) that the enterprises are able to secure from the consumers as rents. Since the consumers in the investment layer clearly prefer more of resource 1 to resource 2 partially due to its lower price, they then would be willing to rent out more of their endowment to enterprise 
1. This is revealed by equation (15) where the price of an enterprise resource is inversely proportional to the amount of input it is able to secure. This is shown by allocation of capital and labour to layer 1 in Fig. 9 . The outputs of enterprises also depend on their technology indices as shown in Fig. 7(a). The results obtained from all the layers have been confirmed to be balance in terms of demand and supply of all resources in all the markets.

\section{Conclusion}

In this study we have presented a decision support model for resource procurement in a convergent supply network. Since the topology of this type of network is such that there are complementary resources, it is impossible to model the entire supply network as a single market where the existence of equilibrium is predicated by the assumption of gross substitutionality of market resources. The approach in this study is to divide the supply network into a number of virtual markets made up of autonomous enterprises. The market value propagation protocol constructed then distributes the network resources based on the private valuation for resources and preference indices for suppliers of individual enterprises. The MOP algorithm executed at each phase of the MVPP reduces the computational efforts required in allocating resources because of its convergence speed as compared with some other competitive general equilibrium algorithms.

The market-based model we have proposed introduces some other opportunities for further research into the resource allocation problem. The estimation of private valuations for endowments of procurement agents and the determination of the preference indices for the procurement agents are interesting subject of future research. While it may not be possible to determine exact values for these variables due to the presence of some factors that are not quantifiable, approximate values will go a long way in improving the quality of results obtained from the model.

\section{参考文献}

[1] H. Stadtler: Supply chain management - an overview; Supply Chain Management and Advanced Planning - Concepts, Models, Software and Case Studies (H. Stadtler and C. Kilger (Eds.)), pp. 9-35, Springer (2005)

[2] M. E. Porter: Competitive Strategy, Techniques for Analyzing Industries and Competitors, The Free Press
(1998)

[3] H. R. Varian: Microeconomic Analysis (Third Edition), W.W. Norton \& Company (1992)

[4] H. Scarf: An example of an algorithm to calculate general equilibrium prices; Cowles Foundation Discussion Paper, No. 276, Cowles Foundation for Research in Economics, Yale University (1969)

[5] M. P. Wellman: A market-oriented programming environment and its application to distributed multicommodity flow problems; Journal of Artificial Intelligence Research, (1), pp. 1-23 (1993)

[6] L. M. Camarinha-Mathos: The virtual enterprise concept; Infrastructures for Virtual Enterprises., Kluwer, pp. 3-14 (1999)

[7] T. Kaihara and S. Fujii: A study on virtual market for optimal mediation in economic society; Experiments in Economic Sciences-New Approaches to Solving Realworld Problems, pp. 494-499 (2004)

[8] J. F. Opadiji and T. Kaihara: A study on resource allocation optimization in a multi-commodity virtual enterprise network; Memoirs of Graduate School of Science and Technology, Kobe University, No. 25A, pp. 7-23, Graduate School of Science and Technology, Kobe (2007)

[9] T. Kaihara, S. Fujii and N. Yoshimura: Validation of pareto-optimality in Walrasian virtual market equilibrium solution; ISCIE Journal, (17), No. 10, pp. 444$450(2004)$

\section{著 者 略 歴}

Jayeola Femi Opadiji

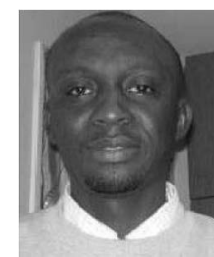

He is presently a doctoral degree candidate in the Systems Design Division of the Graduate School of Science and Technology, Kobe University, Japan. He received his B.Eng. and M.Eng. degrees in Electrical Engineering from University of Ilorin, Ilorin, Nigeria in 2000 and 2004 respectively. His research interests include information technology applications in business and manufacturing systems.

Toshiya Kaihara (Member)

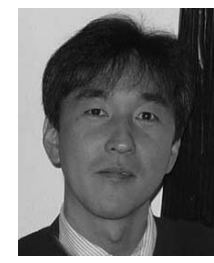

He is a Professor of Graduate School of Engineering at Kobe University, Kobe, Japan. He received B.E and M.E. from Kyoto University, Kyoto, Japan, and Ph.D. from Imperial College, University of London, London, UK. His research interests include modeling, optimization and analysis of production planning, scheduling, logistics and supply chain with sociological multi-agent systems. He is a member of JSPE, SICE, IFIP, IEEE and others. 